\title{
Intestinal microbiota: The hidden gems in the gut?
}

\author{
Pipat Piewngam, ${ }^{1}$ François De Mets, ${ }^{2}$ Michael Otto ${ }^{1}$
}

\begin{abstract}
Within the last decade, our understanding of the role of the intestinal microbiota in health and disease has rapidly increased due to significant advances in next-generation sequencing technologies. Scientists have discovered more and more gut microbes with supposedly "beneficial" roles for human health and are starting to identify the underlying mechanisms. In this review, we summarize the latest knowledge about the human intestinal microbiota, including the intestinal bacteriome, virome and mycobiome. We discuss the function that recent studies attribute to the intestinal microbiota in preventing or controlling selected diseases and present recent research on biotherapeutic approaches to control these diseases.
\end{abstract}

Key words: Intestinal microbiota, Bacteriome, Virome, Mycobiome, Probiotic, Dysbiosis

\footnotetext{
From:

Pathogen Molecular Genetics Section, Laboratory of Bacteriology, National Institute of Allergy and Infectious Diseases, U.S. National Institutes of Health, Maryland, USA

2 Department of Biology, Georgetown University, Washington, D.C., USA
}

\section{Introduction}

The human microbiota is defined as all microorganisms, including a variety of bacteria, fungi, archaea, protists and viruses, found in a defined environment of the human body. ${ }^{1}$ "Microbiome" often refers in a similar way to the microbial organisms that reside in an environmental niche, while strictly speaking this term describes their collective genomes. ${ }^{2,3}$ There have been multiple reports in recent years that describe various ways in which the microbiota influences human health and how the composition of the microbiota can confer resistance to or promote infection by pathogenic organisms. ${ }^{2}$ Major factors that modulate the composition of the gut microbiota in humans include the physical architecture of the gut, diet, lifestyle, several intestinal and non-intestinal diseases, as well as antibiotic consumption and immunosuppressive therapy. ${ }^{4}$ The latter two factors can have a profound impact on microbial diversity by altering the nutritional landscape of the gut, potentially leading to the expansion of pathogenic populations, of which some species can mount severe infections $s^{5-7}$ or cause fatal diseases such as cancer. ${ }^{8}$

Scientists have traditionally sought to identify properties of the human intestinal microbiota by culture-based methods. ${ }^{9}$ Although advances in culturing members of the microbiota that have previously been deemed "unculturable" have been made, these approaches fail to adequately reflect the exceptional diversity of the intestinal microbiota. ${ }^{10}$ For the past decade, however, sequencing-based metagenomics,

\author{
Corresponding author: \\ 1. Pipat Piewngam \\ 50 South Drive, Bethesda, MD 20814, USA \\ E-mail: pipat.piewngam@.nih.gov \\ 2. Michael Otto \\ 50 South Drive, Bethesda, MD 20814, USA \\ E-mail: motto@niaid.nih.gov
}

i.e. the analysis of the metagenomes of genetic material recovered directly from environmental samples in a culture-independent manner, have gained enormous attraction and are now frequently used to study the taxonomic diversity and composition of the intestinal microbiota of both culturable and unculturable microorganisms. ${ }^{11}$ This approach has been material for the recently developed notion that the intestinal microbiota has a substantial and far-reaching impact on human health and disease. In this review, groundbreaking recent studies uncovering the link between human diseases and the intestinal bacteriome, virome and mycobiome will be presented.

\section{The complexity of microbial ecosystems in the human gastrointestinal tract}

The human gastrointestinal (GI) tract is composed of multiple different organs and commonly divided into two areas: the upper and lower GI tract. The upper GI tract includes the mouth, esophagus, stomach, duodenum, jejunum, and ileum, while the lower GI tract includes the colon, rectum, and anus (Figure 1). ${ }^{12}$ The GI tract is considered as the part of the human body with the highest microbial amount and complexity, harboring a large diversity of microorganisms including bacteria, archaea, viruses and fungi (Figure 1). Although rapidly transiting in its characteristics toward the colon, the small 


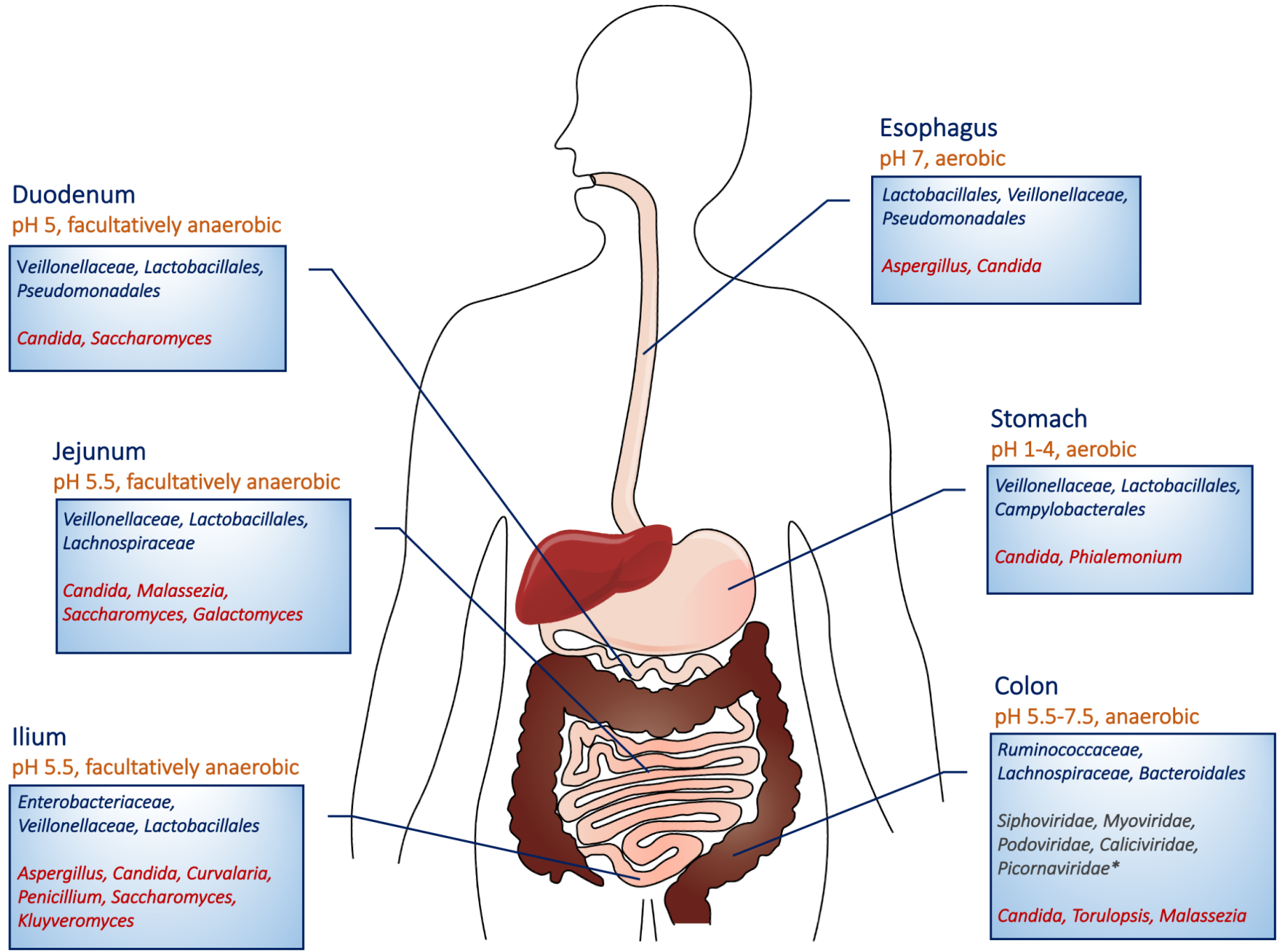

Figure 1. The distribution of bacteria, viruses and fungi among the physiological niches of the human gastrointestinal (GI) tract. The most common bacterial families (blue), viral families (grey) and fungal genus (red) in each GI tract location were presented. ${ }^{\star}$ Intestinal virome in colon were analyzed from fecal sample.

intestine is an inhospitable, harsh environment for microbial life due to high levels of oxygen and the presence of digestive enzymes, bile acids and antimicrobials. ${ }^{12,13}$ The microbiota of the small intestine, which is mainly composed of fast-growing facultative anaerobes, is believed to compete with the host for nutrients. This may be the reason for the lower diversity of the small intestine's microbiota as compared to that of the colon; however, it still contains members from various microbial phyla. ${ }^{13}$ Contrastingly, the large intestine or colon represents a highly favorable niche for microorganisms as a result of a lower concentrations of antimicrobials, slower transit time and absence of digestive secretions. Accordingly, the colon harbors a high microbial diversity and density.

\section{The intestinal bacteriome}

Bacteria are considered the major class of microorganisms contributing to the intestinal microbiota, which is believed to be composed of over 1,800 bacterial genera and over 35,000 different bacterial species. ${ }^{14}$ Bacterial concentration increases gradually from the stomach $\left(10^{1} \mathrm{CFU} / \mathrm{ml}\right)$ to the duodenum $\left(10^{3} \mathrm{CFU} / \mathrm{ml}\right)$, jejunum $\left(10^{4} \mathrm{CFU} / \mathrm{ml}\right)$ and ileum $\left(10^{7} \mathrm{CFU} / \mathrm{ml}\right)$, and culminates in the colon $\left(10^{11}-10^{12} \mathrm{CFU} /\right.$ $\mathrm{ml}) .{ }^{13,15-19}$ The intestinal bacteriome is dominated by four major phyla: Bacteroidetes, Firmicutes, Actinobacteria and Proteobacteria. $^{20-22}$ The Bacteroidetes phylum is composed of anaerobic, bile-resistant, non-spore-forming, Gram-negative rod-shaped bacteria. ${ }^{23}$ Firmicutes is a diverse phylum composed of Gram-positive bacteria distributed over four classes: Bacilli, Clostridia, Erysipelotrichi, and Negativicutes. ${ }^{24}$ Actinobacteria are anaerobic, non-motile, non-spore-forming, Gram-positive rod-shaped bacteria. Lastly, Proteobacteria are aerobic or facultative anaerobic, Gram-negative non-sporeforming rod-shaped bacteria. Firmicutes and Proteobacteria are the major phyla of the small intestine microbiota. However, the abundance of Proteobacteria decreases consistently from the ileum to the colon, whereas Firmicutes, already highly abundant in the small intestine, are still the dominant phylum of bacteria in the colon. ${ }^{18}$ In contrast, Bacteroidetes are present at low levels in the upper GI tract but become a major part of the microbiota in the colon. Generally, the composition of the microbiota markedly changes along the GI tract, with Veillonellaceae, Pseudomonadaceae and Streptococcaceae 
representing the most prevalent bacterial families present in the upper GI tract, while the lower GI tract is dominated by Lachnospiraceae, Bacteroidaceae and Ruminococcaceae. ${ }^{18}$

\section{The intestinal virome}

Over the past decade, most studies on the intestinal microbiota were mainly focused on bacteria. Recently, advances in metagenomics have allowed in-depth analysis also of other microbial communities, especially the intestinal virome. The intestinal virome consists of a diverse collection of DNA and RNA viruses, including eukaryotic viruses, bacteriophages and archaeal viruses. ${ }^{25-28}$ Transmission electron microscopy (TEM), which reveals virus-like particles (VLP), has been used already before the introduction of metagenomics to identify and characterize the intestinal virome. TEM has shown that the human intestinal virome consists mostly of DNA bacteriophages, the viruses that infect bacteria, reaching a number of at least $10^{8}-10^{9}$ VLPs in the intestine. ${ }^{29}$ In these studies, the order Caudovirales along with members of the Myoviridae, Podoviridae, Ackermannviridae and Herelleviridae families have been identified as major constituents of the intestinal virome. ${ }^{29,30}$ More recently, advances in high-throughput sequencing technology overcame the limitation of available tools for the study of the intestinal virome, leading to the discovery of new viral species in the human GI tract. Breitbart et al. reported the first metagenomic analysis of an uncultured viral community from human feces using partial shotgun sequencing, paving the way to a better understanding of the population structure and dynamics of the human intestinal virome. This study reported that the single sample that was analyzed contained about 1,200 different virotypes, with the majority assigned to the Siphoviridae family. ${ }^{31}$

Eukaryotic viruses have also been detected in gut samples from healthy individuals, albeit at very low levels. Anelloviridae, small single-stranded DNA (ssDNA) viruses, are the most common eukaryotic DNA viruses found in the GI tract and several other compartments of the human body. ${ }^{32}$ Based on metagenomic studies, 9 families of eukaryotic DNA viruses have been detected in the GI tract, including Geminiviridae, Herpesviridae, Nanoviridae, Papillomaviridae, Poxviridae, Parvoviridae, Polyomaviridae, Adenoviridae, and Circoviridae. ${ }^{33-35}$ In contrast to DNA viruses, RNA viruses have been largely underappreciated. Caliciviridae, Picobirnaviridae, $\mathrm{Pi}$ cornaviridae, and Reoviridae RNA viruses have been found in gut samples. . $33,36,37^{2}$

\section{The intestinal mycobiome}

Besides bacteria and viruses, many species of fungi have been found in the human GI tract. The number of studies on the intestinal mycobiome and its potential role in human disease is rapidly increasing. It is noteworthy to highlight that specific fungi can modulate the host immune response, resulting in increased risk for immunological disorders. Moreover, the intestinal mycobiome may act as a reservoir for potentially opportunistic pathogens in immunocompromised hosts and may play a role in many diseases that are not obviously related to, or influenced by, the gut. ${ }^{38,39}$ Researchers have traditionally relied on culture-based methods such as microscopy, biochemical assays, and/or growth on selective media to explore the fungal ecosystem in the human GI tract. Although these techniques might seem obsolete, they can provide complementary information. However, the analysis of the intestinal mycobiota by culture-based methods has several limitations due to the problems commonly associated with culturing of fungal microorganisms. ${ }^{40}$ Recent advances in next-generation sequencing technologies and bioinformatics analysis have shed light on the complexity of the intestinal mycobiome, leading to a better understanding of this yet poorly investigated part of the GI microbiota.

The low $\mathrm{pH}$ of the stomach allows only a few acid-tolerant fungi, such as Candida and Phialemonium, to colonize. ${ }^{41}$ Due to the difficulty in collecting samples, our knowledge about the diversity of the mycobiome in the small intestine remains scarce. Early studies of the human intestinal mycobiome, which were based on culturable fungi obtained from intestinal biopsy samples of healthy individuals, revealed that the concentration of intestinal fungi is $\sim 10^{2} \mathrm{CFU} / \mathrm{ml}$ and Candida, notably C. albicans, is the most frequent fungus in the jejunum and ileum. ${ }^{42}$ In a recent clinical study among patients with an intestinal allograft, Saccharomyces cerevisiae and Kluyveromyces waltii were the dominant species in the ileum, while Candida spp., Cryptococcus neoformans, Fusarium oxysporum and Aspergillus clavatus appeared transiently after transplantation. ${ }^{43}$ The mycobiome of the human large intestine and feces is however still largely unknown. Previous studies based on culture-based techniques revealed that Candida, in particular C. albicans, is the most frequent fungus in the colon. ${ }^{42}$

It is widely recognized that the intestinal mycobiome exhibits less diversity than the intestinal bacteriome or virome. Shotgun metagenomics sequencing analysis indicated that the mycobiome only accounts for nearly $0.1 \%$ of the total microorganisms in the intestine. In 2007, Nash et al. investigated the intestinal mycobiome of the Human Microbiome Project (HMP) cohort by sequencing the Internal Transcribed Spacer 2 (ITS2) region and the $18 \mathrm{~S}$ rRNA gene. This study supported the aforementioned notion that the diversity of the intestinal mycobiome is significantly lower than that of the intestinal bacteriome. Saccharomyces (S. cerevisiae), Malassezia (M. restricta), and Candida (C. albicans) were the most prevalent genera. ${ }^{44}$ Several similar studies suggested that Ascomycota is the most predominant phylum in the intestinal niche, followed by the phyla Zygomycota and Basidiomycota. ${ }^{45,46}$ In addition, fungal species belonging to the genera Candida, Cryptococcus, Malassezia, Aspergillus, Saccharomyces, Galactomyces, Trichosporon, and Cladosporium have been found. ${ }^{46}$

\section{Colonization resistance and intestinal dysbiosis}

The intestinal microbiota, particularly anaerobic bacteria, provide for an important host defense mechanism by inhibiting colonization of potentially pathogenic microorganisms both directly and indirectly. This phenomenon, termed "colonization resistance", describes the prevention of overgrowth of indigenous opportunistic pathogens as well as the inhibition of exogenously introduced foreign organisms (Figure 2). ${ }^{47}$ Bacteroides, the dominant genus of the intestinal microbiota, can directly inhibit intestinal pathogens by competing for nutrients or by inducing the production of inhibitory 


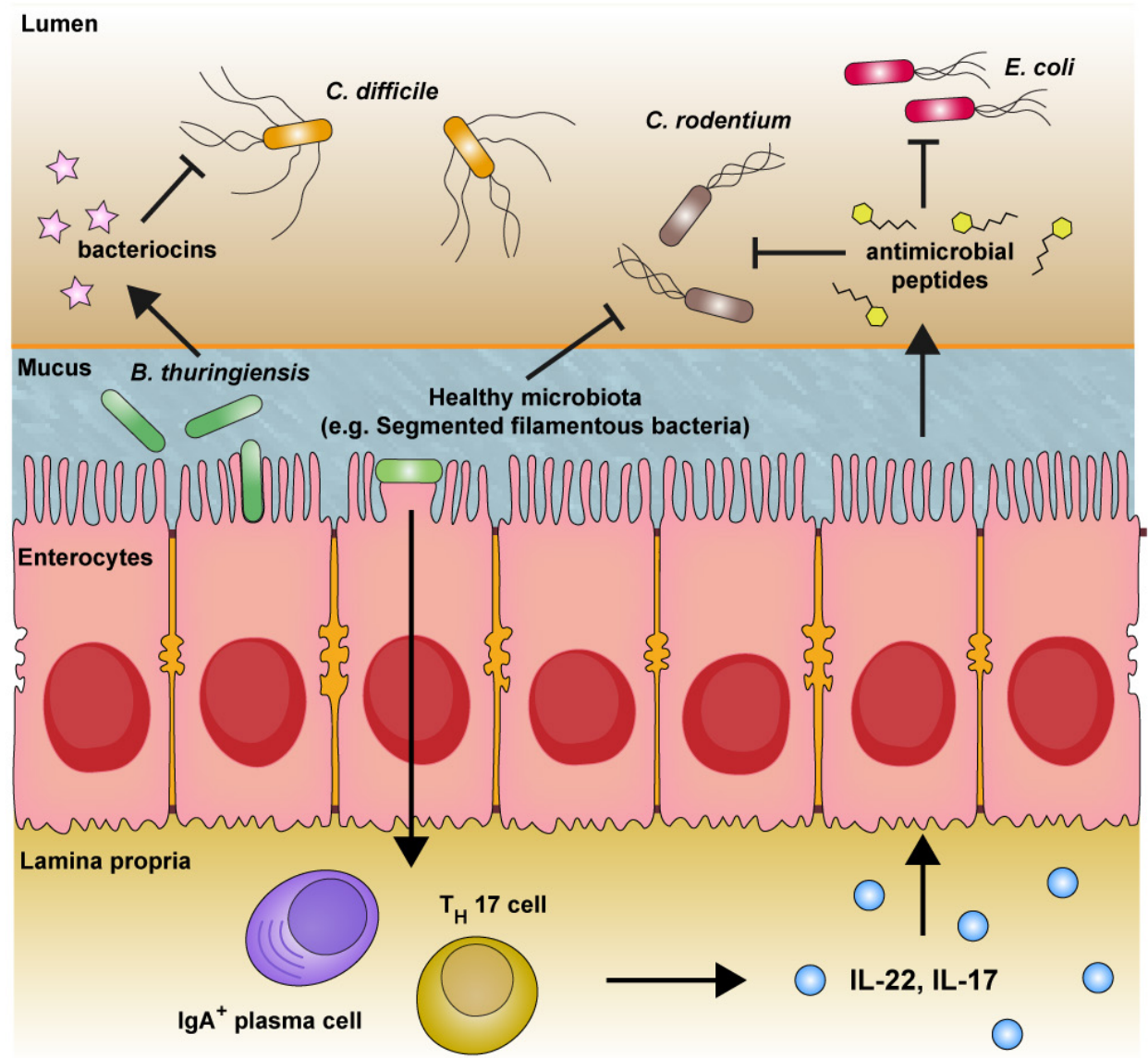

Figure 2. The intestinal microbiota enhances colonization resistance to intestinal pathogens by both indirect (immune-mediated) and direct mechanisms of action. By direct mechanism, Bacteroides thuringiensis can produce bacteriocin that directly targets Clostridium difficile. By indirect mechanism, healthy microbiota, especially segmented filamentous bacteria (SFB), can enhance IgA production, T helper 17 (TH17) cell differentiation, pro-inflammatory cytokine production and epithelial production of antimicrobial peptides which against some Gram-negative bacteria such as Citrobacter rodentium and Escherichia coli.

substances. Bacteroides thetaiotaomicron can consume carbohydrates needed for Citrobacter rodentium, which contributes to the competitive exclusion of this pathogen from the intestinal lumen. Another example involves Porphyromonadaceae family members, which are closely related to Bacteroides spp. and have been associated with protection against Salmonella Typhimurium-induced colitis. ${ }^{48}$

The delicate balance of the intestinal microbiota that leads to colonization resistance can be disrupted by multiple factors such as diet, lifestyle, antibiotic consumption or immunosuppressive therapy, resulting in "intestinal dysbiosis". Intestinal dysbiosis refers to an imbalance in the taxonomical composition and the metagenomic function of the microbial community. Intestinal dysbiosis can be caused by overgrowth of the parts of the intestinal microbiota that have the potential to cause disease, a phenomenon that has been called "bloom of pathobiont", or by the reduction or complete loss of normally residing members of the microbiota, termed "loss of commensal", or both. ${ }^{49}$ Multiple studies have shown that intestinal dysbiosis is associated with health conditions in communicable and non-communicable diseases (Table 1).
However, whether intestinal dysbiosis is a cause or a consequence of specific health conditions or diseases remains an often and controversially discussed issue. By their bacteriostatic and/or bactericidal activities, antibiotics can alter the taxonomic, genomic and functional features of the microbiota. Specifically, antibiotics can significantly decrease the overall amount and diversity of the microbiota, which compromises resistance to colonization by incoming pathogenic bacteria. Furthermore, several non-antibiotic drugs have recently been associated with changes in the gut microbiome composition. ${ }^{50}$

\section{The intestinal microbiota in infectious diseases}

The microbiota that live naturally in the body has frequently been associated with infectious diseases, as it can provide colonization resistance against exogenous or endogenous, opportunistic pathogens. The underlying mechanisms are beginning to be unraveled and are manifold. The role of the intestinal microbiota in infection prevention becomes most clear in individuals that have received broad-spectrum antibiotics, which virtually eliminate the intestinal microbiota. This scenario leads to significantly decreased expression of 
Table 1. Intestinal dysbiosis in health and condition and its possible mechanism

\begin{tabular}{|c|c|c|}
\hline Disease/condition & Change in intestinal microbiota composition & Possible mechanism \\
\hline \multicolumn{3}{|l|}{ Infectious disease } \\
\hline Clostridioides difficile infection (CDI) & $\downarrow \downarrow$ Gram-negative and positive bacteria & $\begin{array}{l}\text { - Inhibition of RegIII } \gamma \text { in small intestine } \\
\text { - Inhibition of bile acid metabolism } \\
\text { - Increasing of } C \text {. difficile spore germination }\end{array}$ \\
\hline Enterococcal bacteremia & $\downarrow \downarrow$ Gram-negative bacteria & - Inhibition of RegIII $\gamma$ in small intestine \\
\hline Chronic hepatitis B virus infection & $\begin{array}{l}\uparrow \uparrow \text { Aspergillus, Candida, Galactomyces, } \\
\text { Saccharomyces, Chaetomium }\end{array}$ & Unknown \\
\hline \multicolumn{3}{|l|}{ Cancer } \\
\hline \multirow[t]{2}{*}{ Colorectal cancer (CRC) } & $\uparrow \uparrow$ enterotoxigenic Bacteroides fragilis & - Deconstruction of E-cadherin by B. fragilis toxin \\
\hline & $\uparrow \uparrow$ Orthobunyavirus, $\downarrow \downarrow$ Mulikevirus & Unknown \\
\hline Pancreatic ductal adenocarcinoma (PDA) & $\uparrow \uparrow$ Malassezia & $\begin{array}{l}\text { - Elevation of tumor progression via MBL cascade } \\
\text { in pancreas by intestinal fungi }\end{array}$ \\
\hline $\begin{array}{l}\text { Immune checkpoint inhibitors (ICIs) } \\
\text { non-responder }\end{array}$ & $\begin{array}{l}\downarrow \downarrow \text { Faecalibacterium, Bifidobacterium longum, } \\
\text { Collinsella aerofaciens, Enterococcus faecium } \\
\text { and Akkermansia muciniphila }\end{array}$ & Unknown \\
\hline \multicolumn{3}{|l|}{ Neurological disorder } \\
\hline Major depressive disorder (MDD) & $\begin{array}{l}\downarrow \downarrow \text { Prevotella, Corprococcus, Dialister } \\
\text { and Faecalibacterium }\end{array}$ & - Low production of microbial $\gamma$-aminobutyric acid \\
\hline Autism spectrum disorders (ASDs) & $\uparrow \uparrow$ Candida & Unknown \\
\hline Rett syndrome & $\uparrow \uparrow$ Candida & Unknown \\
\hline \multicolumn{3}{|l|}{ Gastrointestinal disorder } \\
\hline \multirow[t]{3}{*}{ Inflammatory bowel disease (IBD) } & $\uparrow \uparrow$ Enterobacteriaceae & - Exacerbation of intestinal inflammation \\
\hline & $\begin{array}{l}\downarrow \downarrow \text { Clostridial clusters IV and XIV, B. fragilis } \\
\text { and F. prausnitzii }\end{array}$ & \\
\hline & $\uparrow \uparrow$ Malassezia restricta & $\begin{array}{l}\text { - Elevation of cytokine production from innate cells harbor- } \\
\text { ing the IBD-linked polymorphism in CARD9 }\end{array}$ \\
\hline Irritable bowel syndrome (IBS) & $\begin{array}{l}\uparrow \uparrow \quad \text { Ruminococcaceae and Clostridium cluster } \\
\text { XIVa }\end{array}$ & Unknown \\
\hline
\end{tabular}

antimicrobial molecules in the intestinal mucosa, compromising the intestinal innate immune defense and ultimately leading to increased susceptibility to antibiotic-resistant microbes. The notion that alteration of the intestinal microbiota can increase susceptibility to a variety of infectious diseases has been confirmed in gnotobiotic mice, i.e. mice in which the diverse intestinal microbiota is depleted or reduced to a single bacterial species. ${ }^{51}$ Pultz et al. reported that the anaerobic microbiota in the colon inhibits colonization with vancomycin-resistant enterococci (VRE) by depleting nutrients within cecal contents, limiting the association of VRE with the mucus layer. Moreover, it has been demonstrated that Gram-negative bacteria in the small intestine can produce RegIII $\gamma$, an innate immune effector molecule that kills Gram-positive bacteria, including VRE. ${ }^{5}$ Clostridioides difficile, a Gram-positive anaerobic spore-forming bacterium that can cause $C$. difficile infection (CDI), a leading cause of healthcare-associated morbidity and mortality worldwide, is another prominent example of the importance of niche availability in the pathogenesis of infectious diseases caused by antibiotic-caused depletion of the intestinal microbiota. ${ }^{6}$
Dysbiosis of the mycobiota was shown to affect host immunity by promoting the progression and exacerbation of viral infections such as hepatitis B virus and HIV infections. Chen et al. highlighted that high prevalence of Aspergillus, Candida, Galactomyces, Saccharomyces, and Chaetomium is positively correlated with disease progression in patients with chronic $\mathrm{HBV}$ infection; ${ }^{52}$ and several studies reported that there is high prevalence of Candida in the intestine of HIV-seropositive patients..$^{53-55}$

\section{The intestinal microbiota in cancer}

Various microbial species have been found in cancer tissue. However, the precise roles of the microbiota in cancer development are still unclear and both tumor-suppressive and oncogenic roles of the intestinal microbiota have been described. ${ }^{56}$ Helicobacter pylori is a well-known example of a carcinogenic bacterium and has been studied extensively in the context of gastric cancer. ${ }^{57}$ Interestingly, $H$. pylori infection was found to be significantly correlated with the composition of the fecal microbiota, ${ }^{7}$ although this bacterium lives in the stomach in contrast to most other bacteria, 
which colonize the intestine. Another bacterium whose colonization was found to be associated with intestinal cancer is Fusobacterium nucleatum, which was detected frequently in colorectal cancer patients. ${ }^{11}$ It is not clear whether these organisms are mere opportunists in cancer scenarios, or whether they have on-cogenic or perhaps even tumor development-delaying properties.

Colorectal cancer (CRC) is the second leading cause of death due to cancer, causing about 900,000 deaths worldwide every year. ${ }^{58}$ Western-style diets (high-fat, low fiber, increased meat consumption) are important risk factors for CRC, while the consumption of fiber-rich foods is associated with a lower risk of CRC. ${ }^{59}$ Short-chain fatty acids (SCFAs), such as acetate, butyrate, and propionate, are the main fermentation products of dietary fiber produced by the intestinal microbiota and play an important role in the maintenance of the intestinal immune homeostasis and the protection from inflammation and carcinogenesis. The fecal concentrations of SCFAs are higher in healthy individuals who generally consume lowfat, high-fiber diets, ${ }^{60}$ while recent studies show they decrease in patients with CRC. ${ }^{61}$

High consumption of red and processed meat can also affect the composition of the intestinal microbiota in a way that promotes cancer. Red meat is specifically rich in L-carnitine, which is metabolized by the gut microbiota to trimethylamine (TMA). ${ }^{62}$ TMA is absorbed from the intestine and delivered to the liver and further oxidized by hepatic flavin-dependent monooxygenases (FMOs) to trimethylamine $\mathrm{N}$-oxide (TMAO). ${ }^{63}$ TMAO has been implicated in human disease pathogenesis, including known risk factors for several diseases, particularly cardiovascular disease ${ }^{64}$ In mice, the composition of the intestinal microbiota was altered when L-carnitine was continually fed, resulting in elevated synthesis of TMA. It was also observed that the abundance of $B$. fragilis is significantly higher in individuals consuming a diet rich in beef compared to a vegetarian diet. ${ }^{65} \mathrm{~B}$. fragilis is the least common Bacteroides species in the fecal microbiota but the most frequently isolated from clinical specimens, and can be distinguished by the production of enterotoxins. Intestinal colonization by enterotoxigenic $B$. fragilis (ETBF) is associated with acute diarrheal diseases, inflammatory bowel diseases (IBD), and CRC. ${ }^{66,67}$ B. fragilis toxin destroys the adherens junctions in the intestinal epithelium, which are insured by cell adhesion molecules such as E-cadherin (E-cad). Surface expression of E-cad on epithelial cells plays a central role in preventing cancer, and alteration of E-cad expression has been detected in a large number of cancer cases, which is why E-cad is known as an important tumor suppressor gene. ${ }^{68}$

Besides the consumption of low-fiber, high-fat, and red meat-rich diets, high consumption of alcohol also increases the risk of CRC. ${ }^{69}$ Heavy drinkers have an intestinal microbiota composition that is different from that of non-drinkers and a higher incidence of CRC. Moreover, significant intestinal dysbiosis, for example an unusually high abundance of Proteobacteria,${ }^{70}$ is observed in individuals with alcohol use disorder (AUD). Additionally, Chen et al. have shown that the abundance of Erysipelotrichaceae is increased in the lumen of colorectal cancer patients as compared to healthy controls, which reflects similar results obtained in chronic alcohol-fed mice. ${ }^{71}$
These findings support the notion that CRC development can occur through intestinal dysbiosis by consumption of unhealthy diets or excess alcohol consumption. However, the underlying mechanisms remain to be elucidated.

The intestinal virome has also been associated with cancer. For example, the diversity of the gut bacteriophage community was significantly increased in patients with CRC as compared to controls. ${ }^{72}$ Orthobunyavirus was significantly increased in CRC patients, whereas Mulikevirus was significantly decreased. Additionally, a dysbiotic gut virome was associated with early- and late-stage CRC..$^{72,73}$ Streptococcus bacteriophages were the prevalent phages in early-stage CRC while Parabacteroides phages were the more abundant in latestage CRC. ${ }^{72}$ Whether the observed alteration in the intestinal bacteriophage composition reflects changes within the mucosal bacterial communities remains unclear.

Recently, it has been shown that the mycobiome can accelerate the progression of pancreatic ductal adenocarcinoma (PDA). The mycobiome composition in PDA was found to be distinct from that of the gut or normal pancreas, and the predominant fungal species was Malassezia. The underlying mechanism has been described to include migration of fungi from the gut to the pancreas where they promote tumor progression through activation of the mannan-binding lectin (MBL) cascade. ${ }^{74}$

Notwithstanding these reports, the International Cancer Microbiome Consortium (ICMC) recently stated that there is currently no direct evidence that the human commensal microbiome is a key determinant in the pathogenesis of cancer. ${ }^{75}$ On the contrary, there is evidence to support the notion that the human microbiota, in particular the intestinal microbiota, has a beneficial effect on cancer therapy. Treatment with immune checkpoint inhibitors (ICIs), which have emerged as a novel approach to treat melanoma and other cancers and for example target the cytotoxic T lymphocyte-associated antigen 4 (CTLA-4) or programmed cell death protein 1 (PD-1), was often found to be unsustainable, raising the question whether the intestinal microbiota really consistently promotes anti-tumor immune responses. ${ }^{76-78}$ In one study, the intestinal microbiota of melanoma patients undergoing anti-programmed cell death 1 protein (PD-1) immunotherapy was found to be different between anti-PD-1 therapy responders and non-responders. The responder group showed significantly higher alpha diversity and a significant positive correlation between the number of $\mathrm{CD}^{+} \mathrm{T}$ cells infiltrating the tumor and the abundance of the genus Faecalibacterium, the family Ruminococcaceae, and the order Clostridiales in the gut. ${ }^{76}$ Similarly, Matson et al. demonstrated that composition of commensal bacteria and the clinical response to anti-PD-1 immunotherapy in metastatic melanoma patients were strongly correlated, inasmuch as higher abundance of Bifidobacterium longum, Collinsella aerofaciens, and Enterococcus faecium was observed in responder patients. Furthermore, fecal microbiota transplantation (FMT) from responding patients into germ-free mice improved tumor control via enhancement of T-cell responses, leading to a greater efficacy of anti-programmed cell death protein 1 ligand (PD-L1) therapy. ${ }^{77}$ This major discovery made scientists hypothesize that antibiotic treatment might affect the therapeutic efficacy of ICIs; 
and Routy et al. showed that intestinal dysbiosis induced by antibiotic treatment can affect primary resistance to ICIs in cancer patients. ${ }^{78}$ FMT into germ-free or antibiotic-treated mice from cancer patients responding to ICIs improved the effects of ICIs. Additionally, the abundance of Akkermansia muciniphila increased in PD-1 blockade responders compared to non-responders. Finally, an elevation of the recruitment of $\mathrm{CCR}^{+}{ }^{+} \mathrm{CXCR}^{+}{ }^{+} \mathrm{CD}^{+}{ }^{+} \mathrm{T}$ lymphocytes into mouse tumor beds were observed after oral supplementation with A. muciniphila into non-responder feces-fed mice, which restored the effectiveness of PD-1 blockade. ${ }^{78}$

Although treatment with ICIs targeting CTLA-4 and PD-1/PD-L1 showed promising results in cancer immunotherapy, the treatment can cause serious immune-related adverse effects in some patients, such as colitis. Reconstitution of the intestinal microbiota by FMT elicited ICI-associated colitis, through a relative increase in the proportion of regulatory T-cells within the colonic mucosa. An expansion of $A k$ kermansia, Bifidobacterium and Blautia was also observed in patients after FMT, which has been associated with reduced intestinal inflammation. ${ }^{79}$

\section{The intestinal microbiota in neurological disor- ders}

Links between the intestinal microbiota and the nervous system, in what has been called the microbiota-gut-brain axis, have received increasing recent appreciation. Scientists have started to explore the role of the intestinal microbiota in a broad range of neurological and psychiatric disorders and diseases. For example, antibiotic administration during the first year of life has been correlated with depression and behavioral difficulties later in life. ${ }^{80}$

Major depressive disorder (MDD), a debilitating illness and the second leading global disease, is characterized by depressed mood, diminished interests, impaired cognitive function and vegetative symptoms, such as disturbed sleep or appetite. MDD is associated with $\sim 800,000$ suicidal deaths annually and $20 \%$ of all people are estimated to fulfil the criteria for MDD at some point in their lifetime. ${ }^{81}$ The etiology of MDD is multifactorial and includes genetics, environmental factors, and structural brain alterations as causes. ${ }^{81}$ Recently, MDD has also been associated with the intestinal microbiota. Prevotellaceae, Corprococcus, Dialister and Faecalibacterium were reported to be depleted and Flavonifractor and Bacteroides enterotype 2 to be increased in MDD patients. ${ }^{82}$ Interestingly, the potential of microbes to produce the dopamine metabolite 3,4-dihydroxyphenylacetic acid may correlate positively with mental quality of life and indicates a potential role of microbial $\gamma$-aminobutyric acid production in depression. ${ }^{83}$

Dysbiosis of the intestinal mycobiota has also been reported in neurological diseases. For example, high abundance of intestinal Candida might be associated with the pathogenesis of autism spectrum disorders (ASDs), a group of neurodevelopmental disorders with early-life onset that are characterized by impaired social interactions and communication as well as restricted and repetitive behavior. ${ }^{84}$ Moreover, high relative abundance of intestinal Candida was observed in Rett syndrome patients, a severe and progressive neurological disorder. ${ }^{85}$

\section{The intestinal microbiota in gastrointestinal dis- orders}

Several studies demonstrated that dysregulation of the intestinal microbiota may have detrimental effects on intestinal homeostasis, potentially leading to the development of inflammatory diseases of the digestive tract. Inflammatory bowel disease (IBD), a collective term for a group of intestinal disorders that cause prolonged inflammation of the digestive tract, is often associated with the overgrowth of Enterobacteriaceae. ${ }^{86}$ Intestinal inflammation in IBD that is caused by either environmental or nutritional change may confer a growth advantage to Enterobacteriaceae by providing a more favorable environment for the expansion of especially adherent-invasive E. coli (AIEC) ${ }^{87}$ It has been reported that AIEC strains were found in $21.7 \%$ of ileal samples from patients suffering from Crohn's disease (CD), compared to $6.2 \%$ of healthy controls, and in $36.4 \%$ of neo-terminal ileal samples compared to $22.2 \%$ of controls. ${ }^{88}$ Furthermore, IBD can arise from the reduction of "protective" bacterial species. Several members of the intestinal microbiota can produce metabolites that display anti-inflammatory properties. Thus, when these bacteria are depleted, intestinal inflammation can be exacerbated, resulting in IBD and other intestinal inflammatory diseases. Clostridial clusters IV and XIV, Bacteroides fragilis and Faecalibacterium prausnitzii for example are less abundant in IBD patients compared to normal controls. ${ }^{89}$ In addition, a reduction of Firmicutes has been observed in patients with IBD, with a consequent relative increase of Enterobacteriaceae. Bacteriophages have also been recently shown to play a role in IBD. Bacteriophage numbers are higher at the intestinal mucosal surface and therefore strongly increase during IBD. However, the precise role bacteriophages play in IBD is not yet understood. ${ }^{90}$ Moreover, IBD is associated with changes in the composition of the mycobiota. An increased Basidiomycota/Ascomycota ratio, a decreased proportion of Saccharomyces cerevisiae and an increased proportion of C. albicans were observed in IBD patients compared with healthy controls. ${ }^{91}$ Intestinal colonization with Malassezia restricta, a common skin resident fungus, is higher in CD patients than in controls and also associated with $\mathrm{S} 12 \mathrm{~N}$ polymorphism in the gene encoding CARD9, a signaling adaptor essential for anti-fungal innate immunity. ${ }^{92}$ Exacerbation of colitis observed in $M$. restricta-infected mice is believed to be due to strong cytokine production from innate cells harboring the IBD-linked polymorphism in CARD9.92

Irritable bowel syndrome (IBS) is a functional bowel disorder that commonly causes abdominal pain. IBS pathogenesis has been linked to intestinal dysbiosis based on the finding that an expansion of Ruminococcaceae and Clostridium cluster XIVa was found in IBS patients. ${ }^{93}$ Furthermore, methanogenic microorganisms may play a role in the pathogenesis of IBS, as Methanobrevibacter smithii, a dominant methanogen archaeon in the human gut, was shown to be more abundant in stool samples from patients with constipation-predominant IBS. ${ }^{94}$ Constipation is one of the symptoms often associated 
with IBS and correlated with high methane concentration in the breath. ${ }^{95}$ Finally, intestinal infections with pathogenic enteric bacteria such as Salmonella, Campylobacter, and Shigella, can drive the development of IBS. ${ }^{96}$

\section{Future perspective and conclusion}

The rapidly increasing knowledge on the composition of the microbiota and the mechanisms of interaction between the microorganisms and the host, as well as with each other, are beginning to be exploited to achieve precision editing of the microbiota composition with the goal to lessen disease severity. Scientists have developed several techniques to introduce a beneficial microorganism from healthy donors to patients. FMT, for example, is now widely accepted as a successful rescue treatment for recurrent $C$. difficile infection. However, a recent open-label trial of FMT oral capsules showed that patients experienced bacteremia due to drug-resistant organisms, and there were even fatalities from severe sepsis. ${ }^{97}$ Over the past decade, there has been an increased interest in the use of probiotics to achieve microbiota alteration and a concomitant health benefit to humans. In 2004, the International Scientific Association for Probiotics and Prebiotics (ISAPP) defined probiotics as "live microorganisms that, when administered in adequate amounts, confer a health benefit on the host". ${ }^{8}$ In clinical practice, the number of hospitalized patients receiving probiotics is increasing. ${ }^{99}$ Probiotics have shown benefit in acute infectious diarrhea, antibiotic-associated diarrhea, and ulcerative colitis. ${ }^{100}$ However, despite these successes, there are also recent reports on problems associated with probiotic therapies. Yelin et al. recently reported that probiotic strains can directly cause bacteremia and adaptively evolve within ICU patients. ${ }^{101}$ The efficacy of current probiotic approaches in treatment and prevention is both strain-specific and disease-specific. Moreover, person-specific features, including diet, age and microbiome, also contribute to heterogeneity in the outcome of probiotic supplementations. ${ }^{102}$ The development of a target-specific or person-specific probiotic approach is challenging. This approach could address the heterogeneity inherent to probiotic strains, host and microbiome and serve as "precision probiotic". ${ }^{103} \mathrm{Fi}$ nally, a better assessment of the risks and benefits of probiotics is urgently needed. Generally, more evidence from animal and human studies is required to substantiate the potential of biotherapeutic approaches.

\section{Acknowledgements}

This work was supported by the Intramural Research Program of the National Institute of Allergy and Infectious Diseases (NIAID), U.S. National Institutes of Health (NIH).

\section{References}

1. Sekirov I, Russell SL, Antunes LC, Finlay BB. Gut microbiota in health and disease. Physiol Rev. 2010;90(3):859-904.

2. Clemente JC, Ursell LK, Parfrey LW, Knight R. The impact of the gut microbiota on human health: an integrative view. Cell. 2012;148(6): 1258-70.

3. Backhed F, Fraser CM, Ringel Y, Sanders ME, Sartor RB, Sherman PM, et al. Defining a healthy human gut microbiome: current concepts, future directions, and clinical applications. Cell Host Microbe. 2012;12(5): 611-22.
4. Hall AB, Tolonen AC, Xavier RJ. Human genetic variation and the gut microbiome in disease. Nat Rev Genet. 2017;18(11):690-9.

5. Brandl K, Plitas G, Mihu CN, Ubeda C, Jia T, Fleisher M, et al. Vancomycin-resistant enterococci exploit antibiotic-induced innate immune deficits. Nature. 2008;455(7214):804-7.

6. Buffie CG, Jarchum I, Equinda M, Lipuma L, Gobourne A, Viale A, et al. Profound alterations of intestinal microbiota following a single dose of clindamycin results in sustained susceptibility to Clostridium difficile -induced colitis. Infect Immun. 2012;80(1):62-73.

7. Wang D, Li Y, Zhong H, Ding Q, Lin Y, Tang S, et al. Alterations in the human gut microbiome associated with Helicobacter pylori infection. FEBS Open Bio. 2019;9(9):1552-60.

8. Zitvogel L, Galluzzi L, Viaud S, Vetizou M, Daillere R, Merad M, et al. Cancer and the gut microbiota: an unexpected link. Sci Transl Med. 2015;7(271):271ps1.

9. Rajilic-Stojanovic M, de Vos WM. The first 1000 cultured species of the human gastrointestinal microbiota. FEMS Microbiol Rev. 2014;38(5): 996-1047.

10. Browne HP, Forster SC, Anonye BO, Kumar N, Neville BA, Stares MD, et al. Culturing of 'unculturable' human microbiota reveals novel taxa and extensive sporulation. Nature. 2016;533(7604):543-6.

11. Qin J, Li R, Raes J, Arumugam M, Burgdorf KS, Manichanh C, et al. A human gut microbial gene catalogue established by metagenomic sequencing. Nature. 2010;464(7285):59-65.

12. Greenwood-Van Meerveld B, Johnson AC, Grundy D. Gastrointestinal Physiology and Function. Handb Exp Pharmacol. 2017;239:1-16.

13. Zoetendal EG, Raes J, van den Bogert B, Arumugam M, Booijink CC, Troost FJ, et al. The human small intestinal microbiota is driven by rapid uptake and conversion of simple carbohydrates. ISME J. 2012;6(7): 1415-26.

14. Frank DN, St Amand AL, Feldman RA, Boedeker EC, Harpaz N, Pace NR. Molecular-phylogenetic characterization of microbial community imbalances in human inflammatory bowel diseases. Proc Natl Acad Sci U S A. 2007;104(34):13780-5.

15. Donaldson GP, Lee SM, Mazmanian SK. Gut biogeography of the bacterial microbiota. Nat Rev Microbiol. 2016;14(1):20-32.

16. Sundin $\mathrm{OH}$, Mendoza-Ladd A, Zeng M, Diaz-Arevalo D, Morales E, Fagan BM, et al. The human jejunum has an endogenous microbiota that differs from those in the oral cavity and colon. BMC Microbiol. 2017;17(1):160.

17. Villmones HC, Haug ES, Ulvestad E, Grude N, Stenstad T, Halland A, et al. Species Level Description of the Human Ileal Bacterial Microbiota. Sci Rep. 2018;8(1):4736.

18. Vuik F, Dicksved J, Lam SY, Fuhler GM, van der Laan L, van de Winkel A, et al. Composition of the mucosa-associated microbiota along the entire gastrointestinal tract of human individuals. United European Gastroenterol J. 2019;7(7):897-907.

19. Mailhe M, Ricaboni D, Vitton V, Gonzalez JM, Bachar D, Dubourg G, et al. Repertoire of the gut microbiota from stomach to colon using culturomics and next-generation sequencing. BMC Microbiol. 2018; 18(1):157.

20. Piewngam P, Quinones M, Thirakittiwatthana W, Yungyuen T, Otto M, Kiratisin P. Composition of the intestinal microbiota in extended -spectrum beta-lactamase-producing Enterobacteriaceae carriers and non-carriers in Thailand. Int J Antimicrob Agents. 2019;53(4):435-41.

21. Piewngam P, Zheng Y, Nguyen TH, Dickey SW, Joo HS, Villaruz AE, et al. Pathogen elimination by probiotic Bacillus via signalling interference. Nature. 2018;562(7728):532-7.

22. Eckburg PB, Bik EM, Bernstein CN, Purdom E, Dethlefsen L, Sargent $\mathrm{M}$, et al. Diversity of the human intestinal microbial flora. Science. 2005;308(5728):1635-8.

23. Wexler HM. Bacteroides: the good, the bad, and the nitty-gritty. Clin Microbiol Rev. 2007;20(4):593-621.

24. Galperin MY. Genome Diversity of Spore-Forming Firmicutes. Microbiol Spectr. 2013;1(2).

25. Moreno-Gallego JL, Chou SP, Di Rienzi SC, Goodrich JK, Spector TD, Bell JT, et al. Virome Diversity Correlates with Intestinal Microbiome Diversity in Adult Monozygotic Twins. Cell Host Microbe. 2019;25(2): 261-72 e5.

26. Garmaeva S, Sinha T, Kurilshikov A, Fu J, Wijmenga C, Zhernakova A. Studying the gut virome in the metagenomic era: challenges and perspectives. BMC Biol. 2019;17(1):84. 
27. Mukhopadhya I, Segal JP, Carding SR, Hart AL, Hold GL. The gut virome: the 'missing link' between gut bacteria and host immunity? Therap Adv Gastroenterol. 2019;12:1756284819836620.

28. Neil JA, Cadwell K. The Intestinal Virome and Immunity. J Immunol. 2018;201(6):1615-24.

29. Lepage P, Colombet J, Marteau P, Sime-Ngando T, Dore J, Leclerc M. Dysbiosis in inflammatory bowel disease: a role for bacteriophages? Gut. 2008;57(3):424-5.

30. Hoyles L, McCartney AL, Neve H, Gibson GR, Sanderson JD, Heller KJ, et al. Characterization of virus-like particles associated with the human faecal and caecal microbiota. Res Microbiol. 2014;165(10):803-12.

31. Breitbart M, Hewson I, Felts B, Mahaffy JM, Nulton J, Salamon P, et al. Metagenomic analyses of an uncultured viral community from human feces. J Bacteriol. 2003;185(20):6220-3.

32. Minot S, Bryson A, Chehoud C, Wu GD, Lewis JD, Bushman FD. Rapid evolution of the human gut virome. Proc Natl Acad Sci U S A. 2013;110(30):12450-5.

33. Lim ES, Zhou Y, Zhao G, Bauer IK, Droit L, Ndao IM, et al. Early life dynamics of the human gut virome and bacterial microbiome in infants. Nat Med. 2015;21(10):1228-34.

34. Reyes A, Blanton LV, Cao S, Zhao G, Manary M, Trehan I, et al. Gut DNA viromes of Malawian twins discordant for severe acute malnutrition. Proc Natl Acad Sci U S A. 2015;112(38):11941-6.

35. Wylie KM, Mihindukulasuriya KA, Zhou Y, Sodergren E, Storch GA, Weinstock GM. Metagenomic analysis of double-stranded DNA viruses in healthy adults. BMC Biol. 2014;12:71.

36. Zhang T, Breitbart M, Lee WH, Run JQ, Wei CL, Soh SW, et al. RNA viral community in human feces: prevalence of plant pathogenic viruses. PLoS Biol. 2006;4(1):e3.

37. Kapusinszky B, Minor P, Delwart E. Nearly constant shedding of diverse enteric viruses by two healthy infants. J Clin Microbiol. 2012;50(11): 3427-34.

38. Fasano A, Bove F, Gabrielli M, Petracca M, Zocco MA, Ragazzoni E, et al. The role of small intestinal bacterial overgrowth in Parkinson's disease. Mov Disord. 2013;28(9):1241-9.

39. van Tilburg Bernardes E, Pettersen VK, Gutierrez MW, Laforest-Lapointe I, Jendzjowsky NG, Cavin JB, et al. Intestinal fungi are causally implicated in microbiome assembly and immune development in mice. Nat Commun. 2020;11(1):2577.

40. Nocker A, Burr M, Camper AK. Genotypic microbial community profiling: a critical technical review. Microb Ecol. 2007;54(2):276-89.

41. von Rosenvinge EC, Song Y, White JR, Maddox C, Blanchard T, Fricke WF. Immune status, antibiotic medication and $\mathrm{pH}$ are associated with changes in the stomach fluid microbiota. ISME J. 2013;7(7):1354-66.

42. Cohen R, Roth FJ, Delgado E, Ahearn DG, Kalser MH. Fungal flora of the normal human small and large intestine. N Engl J Med. 1969; 280(12):638-41.

43. Liduma I, Tracevska T, Bers U, Zilevica A. Phenotypic and genetic analysis of biofilm formation by Staphylococcus epidermidis. Medicina (Kaunas). 2012;48(6):305-9.

44. Nash AK, Auchtung TA, Wong MC, Smith DP, Gesell JR, Ross MC, et al. The gut mycobiome of the Human Microbiome Project healthy cohort. Microbiome. 2017;5(1):153.

45. Hoffmann C, Dollive S, Grunberg S, Chen J, Li H, Wu GD, et al. Archaea and fungi of the human gut microbiome: correlations with diet and bacterial residents. PLoS One. 2013;8(6):e66019.

46. Hallen-Adams HE, Suhr MJ. Fungi in the healthy human gastrointestinal tract. Virulence. 2017;8(3):352-8.

47. Lawley TD, Walker AW. Intestinal colonization resistance. Immunology. 2013;138(1):1-11.

48. Buffie CG, Pamer EG. Microbiota-mediated colonization resistance against intestinal pathogens. Nat Rev Immunol. 2013;13(11):790-801.

49. Levy M, Kolodziejczyk AA, Thaiss CA, Elinav E. Dysbiosis and the immune system. Nat Rev Immunol. 2017;17(4):219-32.

50. Maier L, Pruteanu M, Kuhn M, Zeller G, Telzerow A, Anderson EE, et al. Extensive impact of non-antibiotic drugs on human gut bacteria. Nature. 2018;555(7698):623-8.

51. Martin R, Bermudez-Humaran LG, Langella P. Gnotobiotic Rodents: An In Vivo Model for the Study of Microbe-Microbe Interactions. Front Microbiol. 2016;7:409.

52. Chen Y, Chen Z, Guo R, Chen N, Lu H, Huang S, et al. Correlation between gastrointestinal fungi and varying degrees of chronic hepatitis $B$ virus infection. Diagn Microbiol Infect Dis. 2011;70(4):492-8.
53. Gouba N, Drancourt M. Digestive tract mycobiota: a source of infection. Med Mal Infect. 2015;45(1-2):9-16.

54. Uppal B, Kashyap B, Bhalla P. Enteric Pathogens in HIV/AIDS from a Tertiary Care Hospital. Indian J Community Med. 2009;34(3):237-42.

55. Esebelahie NO, Enweani IB, Omoregie R. Candida colonisation in asymptomatic HIV patients attending a tertiary hospital in Benin City, Nigeria. Libyan J Med. 2013;8:20322.

56. Nejman D, Livyatan I, Fuks G, Gavert N, Zwang Y, Geller LT, et al The human tumor microbiome is composed of tumor type-specific intracellular bacteria. Science. 2020;368(6494):973-80.

57. Uemura N, Okamoto S, Yamamoto S, Matsumura N, Yamaguchi S, Yamakido M, et al. Helicobacter pylori infection and the development of gastric cancer. N Engl J Med. 2001;345(11):784-9.

58. Keum N, Giovannucci E. Global burden of colorectal cancer: emerging trends, risk factors and prevention strategies. Nat Rev Gastroenterol Hepatol. 2019;16(12):713-32.

59. Aune D, Chan DS, Lau R, Vieira R, Greenwood DC, Kampman E, et al. Dietary fibre, whole grains, and risk of colorectal cancer: systematic review and dose-response meta-analysis of prospective studies. BMJ. 2011;343:d6617.

60. Ou J, Carbonero F, Zoetendal EG, DeLany JP, Wang M, Newton K, et al. Diet, microbiota, and microbial metabolites in colon cancer risk in rural Africans and African Americans. Am J Clin Nutr. 2013;98(1):111-20.

61. Canani RB, Costanzo MD, Leone L, Pedata M, Meli R, Calignano A. Potential beneficial effects of butyrate in intestinal and extraintestinal diseases. World J Gastroenterol. 2011;17(12):1519-28.

62. Koeth RA, Wang Z, Levison BS, Buffa JA, Org E, Sheehy BT, et al. Intestinal microbiota metabolism of L-carnitine, a nutrient in red meat, promotes atherosclerosis. Nat Med. 2013;19(5):576-85.

63. Janeiro MH, Ramirez MJ, Milagro FI, Martinez JA, Solas M. Implication of Trimethylamine N-Oxide (TMAO) in Disease: Potential Biomarker or New Therapeutic Target. Nutrients. 2018;10(10).

64. Tang WH, Wang Z, Levison BS, Koeth RA, Britt EB, Fu X, et al. Intestinal microbial metabolism of phosphatidylcholine and cardiovascular risk. N Engl J Med. 2013;368(17):1575-84

65. Hentges DJ, Maier BR, Burton GC, Flynn MA, Tsutakawa RK. Effect of a high-beef diet on the fecal bacterial flora of humans. Cancer Res. 1977;37(2):568-71.

66. Sears CL. Enterotoxigenic Bacteroides fragilis: a rogue among symbiotes. Clin Microbiol Rev. 2009;22(2):349-69, Table of Contents.

67. Toprak NU, Yagci A, Gulluoglu BM, Akin ML, Demirkalem P, Celenk T, et al. A possible role of Bacteroides fragilis enterotoxin in the aetiology of colorectal cancer. Clin Microbiol Infect. 2006;12(8):782-6.

68. Jeanes A, Gottardi CJ, Yap AS. Cadherins and cancer: how does cadherin dysfunction promote tumor progression? Oncogene. 2008;27(55):6920-9.

69. Huxley RR, Ansary-Moghaddam A, Clifton P, Czernichow S, Parr CL, Woodward $\mathrm{M}$. The impact of dietary and lifestyle risk factors on risk of colorectal cancer: a quantitative overview of the epidemiological evidence. Int J Cancer. 2009;125(1):171-80.

70. Mutlu EA, Gillevet PM, Rangwala H, Sikaroodi M, Naqvi A, Engen $\mathrm{PA}$, et al. Colonic microbiome is altered in alcoholism. Am J Physiol Gastrointest Liver Physiol. 2012;302(9):G966-78.

71. Yan AW, Fouts DE, Brandl J, Starkel P, Torralba M, Schott E, et al. Enteric dysbiosis associated with a mouse model of alcoholic liver disease. Hepatology. 2011;53(1):96-105.

72. Nakatsu G, Zhou H, Wu WKK, Wong SH, Coker OO, Dai Z, et al Alterations in Enteric Virome Are Associated With Colorectal Cancer and Survival Outcomes. Gastroenterology. 2018;155(2):529-41 e5.

73. Hannigan GD, Duhaime MB, Ruffin MTt, Koumpouras CC, Schloss PD. Diagnostic Potential and Interactive Dynamics of the Colorectal Cancer Virome. mBio. 2018;9(6)

74. Aykut B, Pushalkar S, Chen R, Li Q, Abengozar R, Kim JI, et al. The fungal mycobiome promotes pancreatic oncogenesis via activation of MBL. Nature. 2019;574(7777):264-7.

75. Scott AJ, Alexander JL, Merrifield CA, Cunningham D, Jobin C, Brown R, et al. International Cancer Microbiome Consortium consensus statement on the role of the human microbiome in carcinogenesis. Gut. 2019; 68(9):1624-32.

76. Gopalakrishnan V, Spencer CN, Nezi L, Reuben A, Andrews MC, Karpinets TV, et al. Gut microbiome modulates response to anti-PD-1 immunotherapy in melanoma patients. Science. 2018;359(6371):97-103.

77. Matson V, Fessler J, Bao R, Chongsuwat T, Zha Y, Alegre ML, et al. The commensal microbiome is associated with anti-PD-1 efficacy in metastatic melanoma patients. Science. 2018;359(6371):104-8. 
78. Routy B, Le Chatelier E, Derosa L, Duong CPM, Alou MT, Daillere R, et al. Gut microbiome influences efficacy of PD-1-based immunotherapy against epithelial tumors. Science. 2018;359(6371):91-7.

79. Wang Y, Wiesnoski DH, Helmink BA, Gopalakrishnan V, Choi K, DuPont HL, et al. Author Correction: Fecal microbiota transplantation for refractory immune checkpoint inhibitor-associated colitis. Nat Med. 2019;25(1):188.

80. Slykerman RF, Thompson J, Waldie KE, Murphy R, Wall C, Mitchell EA. Antibiotics in the first year of life and subsequent neurocognitive outcomes. Acta Paediatr. 2017;106(1):87-94.

81. Otte C, Gold SM, Penninx BW, Pariante CM, Etkin A, Fava M, et al. Major depressive disorder. Nat Rev Dis Primers. 2016;2:16065.

82. Sanada K, Nakajima S, Kurokawa S, Barcelo-Soler A, Ikuse D, Hirata A, et al. Gut microbiota and major depressive disorder: A systematic review and meta-analysis. J Affect Disord. 2020;266:1-13.

83. Valles-Colomer M, Falony G, Darzi Y, Tigchelaar EF, Wang J, Tito RY, et al. The neuroactive potential of the human gut microbiota in quality of life and depression. Nat Microbiol. 2019;4(4):623-32.

84. Strati F, Cavalieri D, Albanese D, De Felice C, Donati C, Hayek J, et al. New evidences on the altered gut microbiota in autism spectrum disorders. Microbiome. 2017;5(1):24.

85. Strati F, Cavalieri D, Albanese D, De Felice C, Donati C, Hayek J, et al. Altered gut microbiota in Rett syndrome. Microbiome. 2016;4(1):41.

86. Irving PM, Gibson PR. Infections and IBD. Nat Clin Pract Gastroenterol Hepatol. 2008;5(1):18-27.

87. Boudeau J, Glasser AL, Masseret E, Joly B, Darfeuille-Michaud A. Invasive ability of an Escherichia coli strain isolated from the ileal mucosa of a patient with Crohn's disease. Infect Immun. 1999;67(9):4499-509.

88. Darfeuille-Michaud A, Boudeau J, Bulois P, Neut C, Glasser AL, Barnich $\mathrm{N}$, et al. High prevalence of adherent-invasive Escherichia coli associated with ileal mucosa in Crohn's disease. Gastroenterology. 2004;127(2): 412-21.

89. Kabeerdoss J, Jayakanthan P, Pugazhendhi S, Ramakrishna BS. Alterations of mucosal microbiota in the colon of patients with inflammatory bowel disease revealed by real time polymerase chain reaction amplification of 16S ribosomal ribonucleic acid. Indian J Med Res. 2015;142(1):23-32.

90. Duerkop BA, Kleiner M, Paez-Espino D, Zhu W, Bushnell B, Hassell B, et al. Murine colitis reveals a disease-associated bacteriophage community. Nat Microbiol. 2018;3(9):1023-31.
91. Sokol H, Leducq V, Aschard H, Pham HP, Jegou S, Landman C, et al. Fungal microbiota dysbiosis in IBD. Gut. 2017;66(6):1039-48.

92. Limon JJ, Tang J, Li D, Wolf AJ, Michelsen KS, Funari V, et al. Malassezia Is Associated with Crohn's Disease and Exacerbates Colitis in Mouse Models. Cell Host Microbe. 2019;25(3):377-88 e6.

93. Jeffery IB, O’Toole PW, Ohman L, Claesson MJ, Deane J, Quigley EM, et al. An irritable bowel syndrome subtype defined by species-specific alterations in faecal microbiota. Gut. 2012;61(7):997-1006.

94. Kim G, Deepinder F, Morales W, Hwang L, Weitsman S, Chang C, et al Methanobrevibacter smithii is the predominant methanogen in patient with constipation-predominant IBS and methane on breath. Dig Dis Sci. 2012;57(12):3213-8.

95. Kunkel D, Basseri RJ, Makhani MD, Chong K, Chang C, Pimentel M. Methane on breath testing is associated with constipation: a systematic review and meta-analysis. Dig Dis Sci. 2011;56(6):1612-8.

96. DuPont AW. Postinfectious irritable bowel syndrome. Clin Infect Dis. 2008;46(4):594-9.

97. DeFilipp Z, Bloom PP, Torres Soto M, Mansour MK, Sater MRA Huntley $\mathrm{MH}$, et al. Drug-Resistant E. coli Bacteremia Transmitted by Fecal Microbiota Transplant. N Engl J Med. 2019;381(21):2043-50.

98. Hill C, Guarner F, Reid G, Gibson GR, Merenstein DJ, Pot B, et al Expert consensus document. The International Scientific Association for Probiotics and Prebiotics consensus statement on the scope and appropriate use of the term probiotic. Nat Rev Gastroenterol Hepatol. 2014;11(8):506-14.

99. Yi SH, Jernigan JA, McDonald LC. Prevalence of probiotic use among inpatients: A descriptive study of 145 US hospitals. Am J Infect Control. 2016;44(5):548-53.

100. Suez J, Zmora N, Segal E, Elinav E. The pros, cons, and many unknowns of probiotics. Nat Med. 2019;25(5):716-29.

101. Yelin I, Flett KB, Merakou C, Mehrotra P, Stam J, Snesrud E, et al. Genomic and epidemiological evidence of bacterial transmission from probiotic capsule to blood in ICU patients. Nat Med. 2019;25(11) 1728-32.

102. Sniffen JC, McFarland LV, Evans CT, Goldstein EJC. Choosing an appropriate probiotic product for your patient: An evidence-based practical guide. PLoS One. 2018;13(12):e0209205.

103. Veiga P, Suez J, Derrien M, Elinav E. Moving from probiotics to precision probiotics. Nat Microbiol. 2020;5(7):878-80. 